\title{
Two cases of acute severe flaccid myelitis associated with enterovirus D68 infection in children, Norway, autumn 2014
}

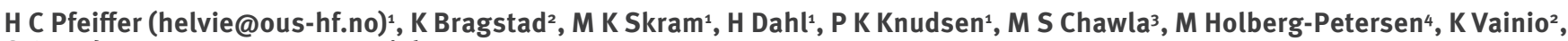
S G Dudman², A M Kran4, A E Rojahn'1

1. Department of Paediatrics, Oslo University Hospital, Oslo, Norway

2. Department of Virology, Norwegian Institute of Public Health, Oslo, Norway

3. Department of Radiology, Oslo University Hospital, Oslo, Norway

4. Department of Microbiology, Oslo University Hospital, University of Oslo, Institute of Clinical Medicine, Oslo, Norway

Enterovirus D68 (EV-D68), phylogenetic clade B was identified in nasopharyngeal specimens of two cases of severe acute flaccid myelitis. The cases were six and five years-old and occurred in September and November 2014. EV-D68 is increasingly associated with acute flaccid myelitis in children, most cases being reported in the United States. Awareness of this possible neurological complication of enterovirus D68 infection is needed.

An unexpectedly high proportion of children were admitted for severe respiratory infections at the Oslo University Hospital, Ullevål, Norway, during September and October, 2014 [1]. Enterovirus was detected in 66 (22\%) of 303 samples from children hospitalised with acute respiratory infection, and in five of 51 samples received from outpatient clinics. Enterovirus D68 (EVD68) was verified in 33 of the enterovirus-positive samples from hospitalised patients, and in one of the outpatients.

We report two cases of severe acute flaccid myelitis (AFM) associated with EV-D68 infection that occurred in September and November 2014 in Norway.

\section{Case 1}

A six year-old girl was referred to a paediatric department in the Oslo area, Norway, on 20 September 2014. She was previously healthy and fully vaccinated according to the Norwegian child vaccination programme, including polio vaccine. She reported a sore throat, neck pain, headache and occasional vomiting for two days. Fever $\left(38.6^{\circ} \mathrm{C}\right)$ occurred on the second day and she became increasingly tired with tachypnoea, coughing and abdominal pain. She presented with a faint voice, reduced general condition, neck pain, but no nuchal rigidity. General and neurological examination was normal. Leucocyte count was $12.9 \times 10^{9}$ cells/L (norm: 5.0-15.5) with neutrophilocytes accounting for $74 \%$. C-reactive protein (CRP) was $4 \mathrm{mg} / \mathrm{L}$ (norm: $0.0-$ 4.0). Viral upper airway infection was suspected, but PCR analysis of a nasopharyngeal specimen was negative for common respiratory viruses, and the patient was discharged. She was readmitted two days later with further deteriorated general condition, general muscle weakness, mainly proximal and more severe in both upper extremities and neck, and weak/ absent deep tendon reflexes. A chest X-ray showed an atelectasis in the left lower lobe but a lower respiratory specimen was not secured. Severe respiratory failure, due to diaphragmatic paresis, resulted in respirator treatment. Meningitis treatment with cefotaxime, ampicillin and aciclovir was started. Acute disseminated encephalomyelitis (ADEM) was suspected, and methylprednisolone instituted.

Cerebrospinal fluid (CSF) pleocytosis $\left(173 \times 10^{6}\right.$ cells/L (norm: $0-4$ ), $94 \%$ mononuclear) and a slightly increased CSF protein concentration $(0.45 \mathrm{~g} / \mathrm{L}$ (norm: 0.000-0.450)) were found on Day 4. On Day 6, before intravenous immunoglobulin infusion or plasmapheresis, only a slight pleocytosis $\left(23 \times 10^{6}\right.$ cells/L) was found, however, an increased IgG index indicated intrathecal IgG production but oligoclonal bands were not detected. CSF cell count had normalised on Day 44 , with CSF protein still slightly increased (0.648 g/L).

PCR analyses of the CSF revealed no intrathecal herpes simplex virus, human herpes virus 6 or 7 , varicellazoster virus, Epstein-Barr virus, cytomegalovirus, human parechovirus or enterovirus. Bacterial culture, including for Listeria monocytogenes, was negative. No intrathecal antibodies against Borrelia burgdorferi were detected. Serological analysis showed no infection with herpes simplex virus, varizella-zoster virus, Epstein-Barr virus, cytomegalovirus, Mycoplasma 
pneumoniae, Borrelia burgdorferi, Cryptococcus neoformans or Mycobacterium tuberculosis. Two faecal specimens, collected on Days 21 and 22, showed no growth of enterovirus. Antibodies associated with autoimmune encephalitis could not be detected.

Magnetic resonance imaging (MRI) on Day 4 showed oedema of mainly grey matter in a longitudinal, transverse pattern, extending from the pedunculi cerebri to the thoracolumbar level. Day 8 there was regression of the oedema, and MRI was normal on Day 44. Electroencephalography (EEG) was normal on Day 3 and Day 16, but compatible with encephalitis/ encephalopathy on Day 5 .

Following reports from the United States (US) and Canada on EV-D68, a nasopharyngeal specimen taken on Day 2 was tested with a generic real-time RT-PCR for enteroviruses targeting the 5' non-coding region, and was positive. Sequencing of the PCR product suggested EV-D68, which was confirmed by a real-time RT-PCR EV-D68 assay (ct value 330 cycles). PCR for EV-D68 was negative in serum (Days 4 and 12), faeces (Day 9) and CSF (Days 4 and 44), and in tracheal secretion (Day 30).

The patient now walks steadily, but head control is poor. Motor impairment is worse proximally and still pronounced in the proximal upper extremities and neck where muscular atrophy is evident. She is partly fed through a gastrostomy tube and speaks with a thin voice.

\section{Case 2}

A five year-old girl was referred to a paediatric department in the Oslo area, Norway, on 13 November 2014. She was previously healthy and fully vaccinated according to the Norwegian child-vaccination programme, including polio vaccine. She had a history of upper airway infection, poor feeding and drinking, and fever up to $39.4{ }^{\circ} \mathrm{C}$ for 12 days. On Day 4 of the illness she complained of neck and back pain. On Day 5 , headaches, abdominal pain and vomiting occurred. On Day 6, she complained of stiffness of the neck. The family doctor found a reduced general condition, mild dehydration, but no nuchal rigidity. On Day 7, weakness occurred in the lower extremities, impairing gait, and the pain and general condition had worsened.

Upon examination at the hospital she was awake and alert, with panting tachypnoea. She was tachycardic (pulse: $161 / \mathrm{min}$ ) and febrile $\left(38.4^{\circ} \mathrm{C}\right)$, other vital signs were normal. Neurological examination was normal. White blood cell count was $12.8 \times 10^{9}$ cells $/ \mathrm{L}$ with $77 \%$ neutrophilocytes. CRP was $10 \mathrm{mg} / \mathrm{L}$. A chest $X$-ray showed a pneumonic infiltrate. PCR analysis of a nasopharyngeal specimen was negative for common respiratory viruses. Erythromycin was instituted and the patient was discharged. PCR of a nasopharyngeal aspirate was negative for Mycoplasma pneumoniae and bacterial culture showed significant growth of Haemophilus influenzae. Lower respiratory samples were not secured. On Day 12, the patient was readmitted with increasing weakness of the left arm, gait difficulties, pain in the neck, left shoulder and both legs, most severely when extended. She had dyspnoea, tachypnoea and panting. Brudzinski's sign was positive and she presented flaccid paralysis grade 1-2 of the left arm as well as weakness of neck muscles, she could not hold her head or walk unsupported. The patient hypoventilated since the diaphragm was partly paretic. Continuous positive airway pressure (CPAP) treatment and cough assist machine were instituted and intravenous immunoglobulin infusion was given.

A lumbar puncture revealed $14 \times 10^{6} \mathrm{cells} / \mathrm{L}$, a protein level of $0.88 \mathrm{~g} / \mathrm{L}$ and glucose of $4.4 \mathrm{mmol} / \mathrm{L}$ (norm: 2.5-4.4). There was no serological evidence of autoimmune encephalitis. EV-D68 was detected in a nasopharyngeal specimen collected at Day 7 , using a generic real-time RT-PCR (ct value 30 cycles), however, culture was negative. EV-D68 was not found in stool (Day 18), serum (Day 15), or CSF (Day 15). CSF cultures (virus and bacteria) (Day 15) were negative. An MRI scan on Day 15 showed cervical central medullary oedema and on Day 20 grey matter oedema at cervical and thoracic level, representing myelitis, as well as radiculitis in the lumbar region. Neurography supported the diagnosis of acute anterior myelitis.

The outcome is with severe paresis and atrophy in the proximal left arm and left upper limb girdle.

\section{Sequence analysis for Case 1}

PCR for sequencing was performed as described in Nix et al. [2]. Sequencing was not possible in for Case 2, due to low amounts of virus and insufficient sample material. The sequence from Case 1 was aligned with the sequences of one reported AFM case from 2014 in France as well as other European strains, including the majority of the 16 Norwegian EV-D68 cases identified in autumn 2014 (Figure) [1].

\section{Discussion}

EV-D68 is mainly associated with respiratory disease and was first isolated in California in 1962 from children with airway infection [3,4]. Until 2009, EV-D68 was rarely isolated [5]. Since then, outbreaks of respiratory disease with EV-D68 have occurred worldwide [6-9]. EV-D68 infections in the Dutch population is increasing [10]. In autumn 2014, EV-D68 was found in $11 \%$ of children hospitalised for airway infection in the Oslo region [1]. Of the 34 patients, 32 were younger than seven years.

During an outbreak of respiratory EV-D68-disease in the US in autumn 2014, nine children presented with AFM; EV-D68 was detected in four of eight nasopharyngeal specimens [11]. From 2 August 2014 to 2 March 2015, the American Centers for Disease Control and Prevention (CDC) verified reports of 115 children in 34 US states who developed AFM. These cases are 


\section{FIGURE}

Phylogenetic cluster analysis of enterovirus D68 partial VP1 sequences (207 nt) from an acute flaccid myelitis case, Norway, September 2014

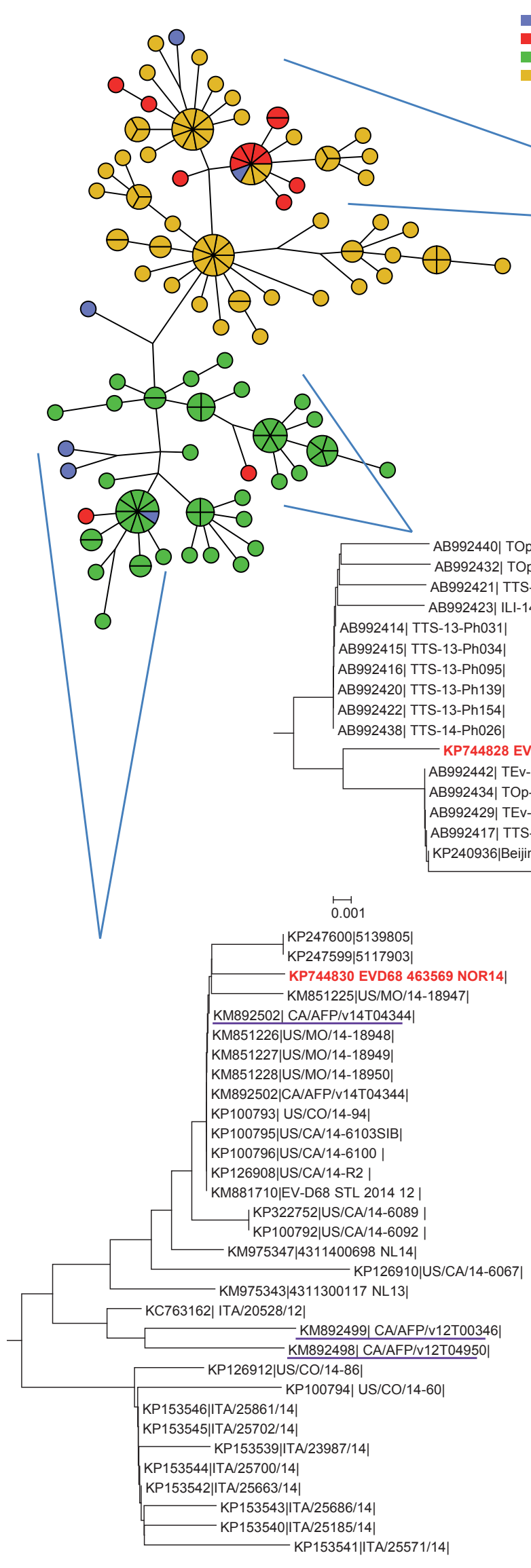

$\stackrel{0.002}{1}$

$\stackrel{\longmapsto}{\longmapsto} 001$

KM851225|US/MO/14-18947|

| CA/AFP/v14T04344|

1251226|US/MO/14-18948

M851227|US/MO/14-1894

100793| US/CO/14-94

81710|EV-D68 STL 201412

KP322752|US/CA/14-6089 |

KP100792|US/CA/14-6092 |

M975343|4311300117 NL13|

KM892499| CA/AFP/v12T0034

\begin{tabular}{|l}
\hline KP153546||TA/25861/14| \\
KP153545||TA/25702/14| \\
KP153539||TA/23987/14| \\
\hline KP153544||ITA/25700/14| \\
KP153542||ITA/25663/14| \\
KP153543||TA/25686/14 \\
KP153540||ITA/25185/14
\end{tabular}

KP153541||TA/25571/14
KC763164| ITA/22516/12|

KF254918|SO9493|

KM975326|3101200440 NL12|

KF254919|SO9320|

KC763169| ITA/24518/12|

Clada B-Subgroup 2

Clada B-Subgroup 1 KF254913|ID72|

KF254920|SO9277|

KM851230|US/IL/14-18952

KC763167| ITA/23695/12 |

KM851229|US/KY/14-18951|

KF254921|SO9411|

KF254923|SO9306

KF254924|SO9406

KF254917|SO9336

KP317480|5876|

N626610|CF267090 FRA14

KP744831 EVD68 463567 NOR14 KP744839 EVD68 466999 NOR14 KM975327|3101200566 NL12

KF254922|SO9288

KP744837 EVD68 466104 NOR14|

KP744826 EVD68 462053 NOR14|

(4311400668 NL14|

-

3101400018 NL14|

KP744825 EVD68 461178 NOR14!

KP744827 EVD68 AFP 462054 NOR14|

KP744829 EVD68 462057 NOR14|

KP744833 EVD68 463572 NOR14

KP744834 EVD68 463573 NOR14|

KP744836 EVD68 464536 NOR1

KM975328|3101300356 NL13

KP744824 EVD68 461177 NOR14

KP744838 EVD68 466311 NOR14

KM975333|3101400474 NL14|

KM975330|3101400307 NL14

KM975350|4311400743 NL14|

KM975334|3101400542 NL14|

KM975331|3101400309 NL14|

$\stackrel{\vdash}{0.002}$

AB

AB 2417 | TTS-13-Ph096|

KP240936|Beijing-R0132|

AB992433| TOp-13-Ph396

Phylogenetic and cluster analysis with currently available EV-D68

sequences in GenBank $(n=231)$. Maximum parsimony clustering

(BioNumerics, Applied Maths) of EV-D68 clade B viruses are

shown together with neighbor-joining (Kimura-model) (Mega v.6)

subtrees containing Norwegian viruses marked in red. Case 1

is marked in purple. Other AFM cases are underlined in purple.

Viruses are named with GenBank accession numbers followed by strain name. 
currently being investigated [12]. A single European case of EV-D68-associated AFM is reported [13]. This virus was of Clade $B$, closely related to Dutch, Spanish and Italian types from 2009 to 2014. From outbreaks in Asia and Australia, enterovirus-71 (EV-A species) is known to cause AFM $[14,15]$. However EV-D68 has rarely been reported to cause AFM, and almost never been isolated from CSF $[5,14]$.

In the two AFM cases we report here, the virus was only detected in the early nasopharyngeal specimens, highlighting the importance of obtaining this specimen early. Pleocytosis, increased intrathecal IgG index, increased CFS-proteins, spinal grey matter-oedema and denervation on neurophysiology may indicate direct CNS infection and neuron destruction. A causal relation is however not proven since no direct evidence of EV-D68 infection in the CNS has been found. Detection of EV-D68-virus may have been coincidental and due to the high incidence of the virus [9]. However, the two cases share striking clinical and imaging similarities [16] and no other pathogen has been detected. Intrathecal EV-D68 antibody detection would be indicative of EV-D68 being the neurotrophic pathogen, however this analysis is not available in our hospital and was therefore not performed.

Sequencing of the VP1 gene obtained from Norwegian cases showed a similar genotype in the AFM case and non-AFM cases. While a statistical increase is difficult to prove for such a rare disease, the authors are of the opinion that there is an increase in AFM cases associated with enterovirus D-68 as has been reported from the US [17]. On basis of our findings we can speculate that this may not be caused by increased neurotrophy in a single genotype, but perhaps due to an increased number of EV-D68-infected individuals. It is possible, however, that mutations in non-VP1 regions are responsible for increased neurotrophy.

As we found an identical VP1-sequence in Case 1 and in a child with solely respiratory disease, host-related factors are likely to be of importance for the individual risk of developing AFM. At least three different EV-D68 strains were circulating in Norway in the autumn of 2014, suggesting three separate introductions. No differences in the partial sequence of the $\mathrm{VP}_{1}$ region were observed between the AFM case and non-AFM cases. International collaboration is needed to confirm the association between EV-D68 and AFM, and to achieve knowledge on treatment and outcome. It is important to recognise this disease, which may become life-threatening.

\section{Acknowledgements}

We are grateful for technical assistance by Pundharika Barkved performing the PCR analysis and sequencing.
Conflict of interest

None declared.

Authors' contributions

HCVP drafted the paper, collected information on the patient, analysed and interpreted it, as well as examined the patient and coordinated the work. KB developed the EV-D68 $P C R$ and sequencing protocol and carried out the sequence analysis followed by the phylogenetic analysis. KV and SGD cultivated virus samples. HCVP, MKS, HMD, PKK, MSC, MHP, $\mathrm{KV}, \mathrm{KB}, \mathrm{SGD}, \mathrm{AMBK}$ and $\mathrm{AER}$ assisted in analysis, interpretation of data for the work, revised it critically for important intellectual content, and final approval of the version to be published. All authors approved the final article.

\section{References}

1. Bragstad K, Jakobsen K, Rojahn AE, Skram MK, Vainio K Holberg-Petersen $M$, et al. High frequency of enterovirus D68 in children hospitalised with respiratory illness in Norway, autumn 2014. Influenza Other Respi Viruses.

2. Nix WA, Oberste MS, Pallansch MA. Sensitive, seminested PCR amplification of VP1 sequences for direct identification of all enterovirus serotypes from original clinical specimens. J Clin Microbiol. 2006;44(8):2698-704. http://dx.doi.org/10.1128/ JCM.00542-06 PMID:16891480

3. Schieble JH, Fox VL, Lennette EH. A probable new human picornavirus associated with respiratory diseases. Am J Epidemiol. 1967;85(2):297-310. PMID:4960233

4. Oberste MS, Maher K, Schnurr D, Flemister MR, Lovchik JC, Peters $\mathrm{H}$, et al. Enterovirus 68 is associated with respiratory illness and shares biological features with both the enteroviruses and the rhinoviruses. J Gen Virol. 2004;85(Pt 9):2577-84. http://dx.doi.org/10.1099/vir.0.79925-0 PMID:15302951

5. Khetsuriani N, Lamonte-Fowlkes A, Oberst S, Pallansch MA; Centers for Disease Control and Prevention. Enterovirus surveillance--United States, 1970-2005. MMWR Surveill Summ. 2006;55(8):1-20. PMID:16971890

6. Hasegawa S, Hirano R, Okamoto-Nakagawa R, Ichiyama T, Shirabe K. Enterovirus 68 infection in children with asthma attacks: virus-induced asthma in Japanese children. Allergy. 2011;66(12):1618-20. http://dx.doi.org/10.1111/j.13989995.2011.02725.x PMID:21958204

7. Tokarz R, Kapoor V, Wu W, Lurio J, Jain K, Mostashari F, et al. Longitudinal molecular microbial analysis of influenza-like illness in New York City, May 2009 through May 2010. Virol J. 2011;8(1):288. http://dx.doi.org/10.1186/1743-422X-8-288 PMID:21658237

8. Imamura T, Okamoto M, Nakakita S, Suzuki A, Saito M, Tamaki R, et al. Antigenic and receptor binding properties of enterovirus 68. J Virol. 2014;88(5):2374-84. http://dx.doi. org/10.1128/JVI.03070-13 PMID:24371050

9. Meijer A, Benschop KS, Donker GA, van der Avoort HG Continued seasonal circulation of enterovirus D68 in the Netherlands, 2011-2014. Euro Surveill. 2014;19(42):20935 http://dx.doi.org/10.2807/1560-7917.ES2014.19.42.20935 PMID:25358039

10. Poelman R, Schölvinck EH, Borger R, Niesters HG, van Leer-Buter C. The emergence of enterovirus D68 in a Dutch University Medical Center and the necessity for routinely screening for respiratory viruses. J Clin Virol. 2015;62:1-5. http://dx.doi.org/10.1016/j.jcv.2014.11.011 PMID:25542461

11. Pastula DM, Aliabadi N, Haynes AK, Messacar K, Schreiner T, Maloney J, et al.; Centers for Disease Control and Prevention (CDC). Acute neurologic illness of unknown etiology in children - Colorado, August-September 2014. MMWR Morb Mortal Wkly Rep. 2014;63(40):901-2. PMID:25299607

12. Centers for Disease Prevention and Control (CDC). Summary of Findings: Investigation of Acute Flaccid Myelitis in U.S. Children, 2014. Atlanta: CDC. [Accessed: 5 March 2015]. Available from: http://www.cdc.gov/ncird/investigation/viral/ sep2014/investigation.html

13. Lang M, Mirand A, Savy N, Henquell C, Maridet S, Perignon $R$, et al. Acute flaccid paralysis following enterovirus D68 associated pneumonia, France, 2014. Euro surveillance: bulletin Europeen sur les maladies transmissibles = European communicable disease bulletin. 2014;19(44). 
14. Kreuter JD, Barnes A, McCarthy JE, Schwartzman JD,

Oberste MS, Rhodes $\mathrm{CH}$, et al. A fatal central nervous

system enterovirus 68 infection. Arch Pathol Lab Med.

2011;135(6):793-6. PMID:21631275

15. McMinn P, Stratov I, Nagarajan L, Davis S. Neurological

manifestations of enterovirus 71 infection in children during an outbreak of hand, foot, and mouth disease in Western Australia. Clin Infect Dis. 2001;32(2):236-42. http://dx.doi. org/10.1086/318454 PMID:11170913

16. Maloney JA, Mirsky DM, Messacar K, Dominguez SR, Schreiner T, Stence NV. MRI Findings in Children with Acute Flaccid Paralysis and Cranial Nerve Dysfunction Occurring during the 2014 Enterovirus D68 Outbreak. AJNR Am J Neuroradiol. 2015;36(2):245-50. http://dx.doi.org/10.3174/ajnr.A4188 PMID:25414005

17. Messacar K, Schreiner TL, Maloney JA, Wallace A, Ludke J, Oberste MS, et al. A cluster of acute flaccid paralysis and cranial nerve dysfunction temporally associated with an outbreak of enterovirus D68 in children in Colorado, USA. Lancet. 2015;pii: S0140-6736(14)62457-o. http://dx.doi. org/10.1016/S0140-6736(14)62457-0. 